\title{
Energy-Momentum Distribution of Six-Dimensional Geometric Model of Gravitational Field
}

\author{
Sarfraz Ali ${ }^{1 *}$ and M. Jamil Amir ${ }^{2}$ \\ ${ }^{1}$ Department of Mathematics, University of Education Lahore, Faisalabad Campus, Pakistan \\ ${ }^{2}$ Department of Mathematics, Government Post Graduate, College (Boys), Taunsa, DG Khan, Pakistan \\ *Corresponding author
}

\author{
Article Info \\ Keywords: General Relativity, Six Di- \\ mensional Geometric Model, \\ Gravitational Field, Energy and Mo- \\ mentum. \\ 2010 AMS: $83 C x x$ \\ Received: 12 April 2019 \\ Accepted: 8 August 2019 \\ Available online: 30 September 2019
}

\begin{abstract}
Much work has been done in exploring the energy-momentum distribution of different four-dimensional spacetimes using different prescriptions. In this paper, we intend to explore the energy and momentum density of six-dimensional geometric model of the gravitational field. The model was constructed by postulating a six-dimensional spacetime manifold with a structure of spacetime of absolute parallelism. For this purpose, we consider the metric representing the geometric model and use five prescriptions, namely, Einstein, Landau-Lifshitz, Bergmann-Thomson, Papapetrou, and Möller in the framework of General Relativity. The energy and momentum turn out to be well defined and finite. The comparison of the results shows that Einstein and Bergmann-Thomson prescriptions yield same energy-momentum densities but different from the other three prescriptions. It is mentioning here that the energy vanishes in the case of Möller's prescription and the momentum densities become zero in all the cases.
\end{abstract}

\section{Introduction}

The theory of General Relativity (GR) is recognized as the best among all theories of gravitation, available in the literature, because many important features of the universe have been verified experimentally in the framework of this theory. Although, the issue of localization of energy and momentum is most divisive and longstanding. Huge efforts have been made to resolve this problem by the big names of Physics and Mathematics. Einstein [1] is an innovator who introduced his energy-momentum complexes for the localization of energy and momentum. By the passage of time, a number of efficient scientists, including Landau-Lifshitz [2], Møller [3], Bergmann-Thomson [4], Weinberg [5], Papapetrou [6] and Tolman [7], presented their own energy-momentum complexes. These prescriptions yield feasible results only if one uses cartesian coordinates except Møller's prescription, which is independent of coordinate choice in case of energy only.

Misner and Sharp [8] proved that the energy can be localized in the spherical coordinate system. Cooperstock and Sarracino [9] proved that if the energy can be localized in a spherically symmetric coordinate system then it surely be localized in non-spherical symmetric coordinate which are static or quasistatic. Virbhadra and his coworkers $[10,11]$ investigated the energy-momentum distribution of different spacetimes, such as the Kerr-Newmann, Kerr-Schild classes, Einstein-Rosen, Vaidya, and Bonnor-Vaidya spacetimes. They showed that different energy-momentum prescriptions could give the same consequences, which are coincident with the results obtained by Tod [12] using the Penrose [13] formalism in the context of quasi-local mass.

Various scientist $[14,15]$ described the energy of the gravitational field by presenting the Hamiltonian approach in the structure of Schwinger's condition [16]. Using this formalism, energy can be determined by an integral of a scalar density in the form of total divergence which appears as the Hamiltonian constraints of this theory. Initially, the problem of localization of energy-momentum emerged in electromagnetism and then it became a serious issue in GR due to the non-tensorial quantities. The localization of energy is discussed in some other theories such as the $f(R)$ theory and Teleparallel (TP) theory of gravity.

Mikhail et al. [17] defined the superpotential in the Møller's tetrad theory which has been used to find the energy in the teleparallel theory of gravity (TTG). Vargas [18] defined the TP version of Bergman, Einstein, and Landau-Lifshitz prescriptions and found that the total energy of the closed Friedman-Robertson-Walker universe is zero by using the last two prescriptions. This agrees with the results of GR available in 
literature [12, 13]. Later, many authors [19]-[23] used TP version of these prescriptions and showed that energy may be localized in TTG. Sharif and Jamil [24]-[32] explored the energy of different spcetimes in GR and TTG and obtained interesting results.

Multamäki et al. [33] generalized the Landau-Lifshitz energy-momentum prescription in $f(R)$ theory of gravity. They also found the energy density for the Schwarzschild de Sitter spacetime. By using generalized Landau-Lifshitz prescriptions, Sharif and Farasat [34] calculated the energy density of plane-symmetric static metric and cosmic string spacetime. Faraoni and Nadeau [35] have discussed some important $f(R)$ models along with their stability conditions. Jamil and his collaborators $[36,37]$ obtained the spatially homogeneous rotating solutions and locally rotating spacetimes in $f(R)$ gravity and the energy contents are obtained for non-trivial solution for particular $f(R)$ model.

Silva et al. [38] proposed a six-dimensional string-like braneworld built from a warped product between a 3-brane and the Hamilton cigar soliton space. They discussed the effects of the evolution of the transverse space on the geometric and physical quantities. The gravitational massless mode remains trapped to the brane and the width of the model depends on the evolution parameter. For the Kaluza-Klein modes, the asymptotic spectrum of mass is the same as for the thin string-like brane and the analog Schrödinger potential also changes according to the flow. Linch and Tartaglino-Mazzucchelli [39] introduced a superspace result for $N=(1,0)$ conformal supergravity in six dimensions. They formulated a locally supersymmetric and super-Weyl invariant action principle in projective superspace.

Popov [40] constructed a six-dimensional pure geometric model by postulating a six-dimensional spacetime manifold with a structure of spacetime of absolute parallelism. He established a clear relation between the Schwarzschild solution of the gravitational field of a point mass and the field of point source torsion. In the case of Teleparallel Equivalent of General Relativity (TEGR), it is equivalent to interpret the gravitation either in terms of curvature or in terms of torsion. Therefore, we try to explore the energy-momentum distribution of six-dimensional teleparallel solution by using GR prescriptions. Our aim is to explore the energy-momentum distribution of this geometric model using different complexes in the context of GR.

\section{Energy-Momentum Distribution of Six Dimensional Geometric Model of Gravitational Field}

We consider the line element is given in Eq.(14) of [40] representing the geometric model of a gravitational field in six dimensions, given as

$$
\begin{aligned}
d s^{2} & =\frac{-\left(3 y_{1}+a^{3}\right)^{-4 / 3}}{1-a /\left(3 y_{1}+a^{3}\right)^{1 / 3}} d y_{1}^{2}-\frac{\left(3 y_{1}+a^{3}\right)^{2 / 3}}{1-y_{2}^{2}} d y_{2}^{2}-\left(3 y_{1}+a^{3}\right)^{2 / 3}\left(1-y_{2}\right)^{2} d y_{3}^{2} \\
& +\left(1-\frac{a}{\left(3 y_{1}+a^{3}\right)^{1 / 3}}\right) d y_{4}^{2}+\frac{1}{2 y_{5}+\gamma} d y_{5}^{2}+\left(2 y_{5}+\gamma\right) d y_{6}^{2}
\end{aligned}
$$

Here, $y_{1}$ stands for time component and rest are the space components.

The Einstein energy-momentum prescription is defined as [1]

$$
\Theta_{a}^{b}=\frac{1}{16 \pi} H_{a, c}^{b c}
$$

where $H^{b c}{ }_{a}$ depends on metric tensor and its first order derivative, found by Freud [42] given as

$$
H_{a}^{b c}=\frac{g_{a d}}{\sqrt{-g}}\left[-g\left(g^{b d} g^{c e}-g^{c d} g^{b e}\right)\right]_{e} .
$$

Here, the term $\Theta_{0}^{0}$ stands for energy density, $\Theta_{i}^{0}(i=1, \ldots, 5)$ are the components of momentum density and the current density components are denoted by $\Theta_{0}^{i}$. Using Eq.(1) in Eq.(3), the non-vanishing components of $H^{b c}{ }_{a}$ turn out to be

$$
\begin{aligned}
& H^{01}{ }_{0}=-H^{10}{ }_{0}=4\left(a-\left(a^{3}+3 y_{1}\right)^{1 / 3}\right), \\
& H^{02}{ }_{0}=-H^{20}{ }_{0}=H^{12}{ }_{1}=-H^{21}{ }_{1}=\frac{2 y_{2}}{\left(a^{3}+3 y_{1}\right)^{2 / 3}}, \\
& H^{04}{ }_{0}=H^{14}{ }_{1}=H_{2}^{24}=H^{35}{ }_{3}=2, \\
& H^{40}{ }_{0}=H^{41}{ }_{1}=H^{42}{ }_{2}=H^{43}{ }_{3}=-2, \\
& H^{12}{ }_{2}=H^{13}{ }_{3}=-H^{21}{ }_{2}=-H^{31}{ }_{3}=-a+2\left(a^{3}+3 y_{1}\right)^{1 / 3}, \\
& H^{41}{ }_{4}=H^{51}{ }_{5}=-H^{14}{ }_{4}=-H^{15}{ }_{5}=3 a-4\left(a^{3}+3 y_{1}\right)^{1 / 3}, \\
& H^{24}{ }_{4}=-H^{42}{ }_{4}=H^{52}{ }_{5}=-H^{25}{ }_{5}=\frac{2 y_{2}}{\left(a^{3}+3 y_{1}\right)^{2 / 3}} .
\end{aligned}
$$

Making use of these values in Eq.(2), the energy and momentum densities of Einstein's prescription turn out to be

$$
\begin{aligned}
& \Theta^{00}=\frac{-3 y_{1}+a\left(a^{2}+a\left(a^{3}+3 y_{1}\right)^{1 / 3}+\left(a^{3}+3 y_{1}\right)^{2 / 3}\right)}{24 \pi y_{1}\left(a^{3}+3 y_{1}\right)^{2 / 3}}, \\
& \Theta^{0 i}=0, \quad(i=1,2, \ldots, 5) .
\end{aligned}
$$

The Landau-Lifshitz's prescription, in GR, is given as [2]

$$
L^{a b}=\frac{1}{16 \pi} l^{a c b d}, c d
$$


where $l^{a b c d}$ is a 4 rank tensor, given as

$$
l^{a b c d}=(-g)\left(g^{a b} g^{c d}-g^{a d} g^{c b}\right) .
$$

The term $L^{00}$ yields the energy density component of the whole system and $L^{i 0}(i=1, \ldots, 5)$ represents the momentum density components. In view of Eq.(1), the Eq.(5) gives the following non-vanishing components of the tensor $l^{a b c d}$

$$
\begin{aligned}
& l^{1001}=l^{0110}=-l^{1010}=-l^{0101}=\left(a^{3}+3 y_{1}\right)^{4 / 3}, \\
& l^{2002}=l^{0220}=-l^{2020}=-l^{0202}=\frac{1-y_{2}^{2}}{-a\left(a^{3}+3 y_{1}\right)^{1 / 3}+\left(a^{3}+3 y_{1}\right)^{2 / 3}}, \\
& l^{3003}=l^{0330}=-l^{3030}=-l^{0303}=\frac{-a\left(a^{3}+3 y_{1}\right)^{1 / 3}+\left(a^{3}+3 y_{1}\right)^{2 / 3}}{\left(a^{3}+3 y_{1}\right)^{1 / 3}\left(-a+\left(a^{3}+3 y_{1}\right)^{1 / 3}\right)\left(-1+y_{2}^{2}\right)}, \\
& l^{4004}=l^{0440}=-l^{4040}=-l^{0404}=-\frac{\left(a^{3}+3 y_{1}\right)^{1 / 3}\left(\gamma+2 y_{4}\right)}{-a+\left(a^{3}+3 y_{1}\right)^{1 / 3}}, \\
& l^{5005}=l^{0550}=-l^{5050}=-l^{0505}=\frac{-a+\left(a^{3}+3 y_{1}\right)}{\left(1-\frac{a}{\left(a^{3}+3 y_{1}\right)^{1 / 3}}\right)\left(\gamma+2 y_{4}\right)}, \\
& l^{2112}=l^{1221}=-l^{2121}=-l^{1212}=-\left(a^{3}+3 y_{1}\right)^{1 / 3}\left(\left(a^{3}+3 y_{1}\right)^{1 / 3}-a\right)\left(y_{2}^{2}-1\right) \text {, } \\
& l^{3113}=l^{1331}=-l^{3131}=-l^{1313}=-\frac{a\left(a^{3}+3 y_{1}\right)^{1 / 3}-\left(a^{3}+3 y_{1}\right)^{2 / 3}}{y_{2}^{2}-1}, \\
& l^{4114}=l^{1441}=-l^{4141}=-l^{1414}=\left(a^{3}+3 y_{1}\right)\left(-a+\left(a^{3}+3 y_{1}\right)^{1 / 3}\right)\left(\gamma+2 y_{4}\right) \text {, } \\
& l^{5115}=l^{1551}=-l^{5151}=-l^{1515}=\frac{\left(a^{3}+3 y_{1}\right)\left(-a+\left(a^{3}+3 y_{1}\right)^{1 / 3}\right)}{\gamma+2 y_{4}}, \\
& l^{3223}=l^{2332}=-l^{3232}=-l^{2323}=\frac{-1}{\left(a^{3}+3 y_{1}\right)^{4 / 3}}, \\
& l^{4224}=l^{2442}=-l^{4242}=-l^{2424}=-\frac{\left(-1+y_{2}^{2}\right)\left(\gamma+2 y_{4}\right)}{\left(a^{3}+3 y_{1}\right)^{2 / 3}}, \\
& l^{5225}=l^{2552}=-l^{5252}=-l^{2525}=\frac{-1+y_{2}^{2}}{\left(a^{3}+3 y_{1}\right)^{2 / 3}\left(\gamma+2 y_{4}\right)}, \\
& l^{4334}=l^{3443}=-l^{4343}=-l^{3434}=-\frac{\gamma+2 y_{4}}{\left(a^{3}+3 y_{1}\right)^{2 / 3}\left(-1+y_{2}^{2}\right)}, \\
& l^{5335}=l^{3553}=-l^{5353}=-l^{3535}=\frac{\left(a^{3}+3 y_{1}\right)^{2 / 3}\left(-1+y_{2}^{2}\right)}{\left(a^{3}+3 y_{1}\right)^{2 / 3}\left(-1+y_{2}^{2}\right)\left(\gamma+2 y_{4}\right)}, \\
& l^{5445}=l^{4554}=-l^{5454}=-l^{4545}=-1 .
\end{aligned}
$$

When we use these values of $l^{a b c d}$ in Eq.(4), the energy and momentum density components of Landau-Lifshitz's prescription are obtained as

$$
\begin{aligned}
L^{00} & =\frac{2 a-\left(a^{3}+3 y_{1}\right)^{1 / 3}}{8 a^{3} \pi+24 \pi y_{1}-8 a \pi\left(a^{3}+3 y_{1}\right)^{2 / 3}} . \\
L^{0 i} & =0, \quad(i=1, \ldots, 5) .
\end{aligned}
$$

In case of Bergmann-Thomson prescription, the energy-momentum density components are defined as [4]

$$
B^{a b}=\frac{1}{16 \pi} M^{a b c}, c
$$

where

$$
M^{a b c}=g^{a d} V_{d}^{b c},
$$

and

$$
V_{d}^{b c}=\frac{g_{d e}}{\sqrt{-g}}\left[-g\left(g^{b e} g^{c f}-g^{c e} g^{b f}\right)\right], f
$$

Here, $B^{00}$ represents the energy density of the entire system and $B^{0 i}(i=1, \ldots, 5)$ stands for the momentum density components. Using Eq.(1) in Eq.(8), we get the following non-vanishing components of $V^{a b}{ }_{c}$

$$
\begin{aligned}
& V_{0}^{01}=-V_{0}^{10}=4\left(a-\left(a^{3}+3 y_{1}\right)^{1 / 3}\right), \\
& V_{0}^{02}=-V_{0}^{20}=V^{12}{ }_{1}=-V^{21}{ }_{1}=\frac{2 y_{2}}{\left(a^{3}+3 y_{1}\right)^{2 / 3}} \text {, } \\
& V_{0}^{04}=V_{1}^{14}=V^{24}{ }_{2}=V_{3}^{34}=2 \text {, } \\
& V_{0}^{40}=V_{1}^{41}=V_{2}^{42}=V_{3}^{43}=-2 \text {, } \\
& V^{12}{ }_{2}=V^{13}{ }_{3}=-V_{2}^{21}=-V^{31}=-a+2\left(a^{3}+3 y_{1}\right)^{1 / 3} \text {, } \\
& V^{41}{ }_{4}=V^{51}{ }_{5}=-V_{4}^{14}=-V^{15}=3 a-4\left(a^{3}+3 y_{1}\right)^{1 / 3} \text {, } \\
& V_{4}^{24}=-V_{4}^{42}=V^{52}=-V^{25}=-\frac{2 y_{2}}{\left(a^{3}+3 y_{1}\right)^{2 / 3}} \text {. }
\end{aligned}
$$

Substituting these values in Eq.(7) and then in Eq.(6), the energy and momentum components of Bergmann-Thomson's prescription turn out to be 


$$
\begin{aligned}
& B^{00}=\frac{-3 y_{1}+a\left(a^{2}+a\left(a^{3}+3 y_{1}\right)^{1 / 3}+\left(a^{3}+3 y_{1}\right)^{2 / 3}\right)}{24 \pi y_{1}\left(a^{3}+3 y_{1}\right)^{2 / 3}} . \\
& B^{0 i}=0, \quad(i=1, \ldots, 5) .
\end{aligned}
$$

Now, the energy-momentum prescription of Papapetrou [6] is given as

$$
\Omega^{a b}=\frac{1}{16 \pi} N^{a b c d}, c d
$$

where

$$
N^{a b c d}=\sqrt{-g}\left(g^{a b} \eta^{c d}-g^{a c} \eta^{b d}+g^{c d} \eta^{a b}-g^{b d} \eta^{a c}\right) .
$$

Here, $\eta^{a b}$ is the metric tensor of Minkowski spacetime. The $\Omega^{00}$ and $\Omega^{0 i}$ represent the energy and momentum density components respectively. The non-vanishing components of $N^{a b c d}$ are evaluated as

$$
\begin{aligned}
& \mathrm{N}^{1010}=\mathrm{N}^{0101}=\frac{1}{1-\frac{a}{\left(a^{3}+3 y_{1}\right)^{1 / 3}}}-\left(a^{3}+3 y_{1}\right)\left(-a+\left(a^{3}+3 y_{1}\right)^{1 / 3}\right) \\
& =-\mathrm{N}^{0011}=-\mathrm{N}^{1100}, \\
& \mathrm{~N}^{2020}=\mathrm{N}^{0202}=-\mathrm{N}^{0022}=-\mathrm{N}^{2200}=\frac{1}{1-\frac{a}{\left(a^{3}+3 y_{1}\right)^{1 / 3}}}+\frac{-1+y_{2}^{2}}{\left(a^{3}+3 y_{1}\right)^{2 / 3}}, \\
& \mathrm{~N}^{3030}=\mathrm{N}^{0303}=-\frac{1}{1-\frac{a}{\left(a^{3}+3 y_{1}\right)^{1 / 3}}}+\frac{1}{\left(a^{3}+3 y_{1}\right)^{2 / 3}\left(-1+y_{2}^{2}\right)} \\
& =-\mathrm{N}^{0033}=-\mathrm{N}^{3300} \\
& \mathrm{~N}^{4040}=\mathrm{N}^{0404}=-\mathrm{N}^{0044}=-\mathrm{N}^{4400}=\gamma-\frac{1}{1-\frac{a}{\left(a^{3}+3 y_{1}\right)^{1 / 3}}}+2 y_{4}, \\
& \mathrm{~N}^{5050}=\mathrm{N}^{0505}=-\mathrm{N}^{0055}=-\mathrm{N}^{5500}=-\frac{1}{1-\frac{a}{\left(a^{3}+3 y_{1}\right)^{1 / 3}}}+\frac{1}{\gamma+2 y_{4}}, \\
& \mathrm{~N}^{2121}=\mathrm{N}^{1212}=\left(a^{3}+3 y_{1}\right)\left(-a+\left(a^{3}+3 y_{1}\right)^{1 / 3}\right)+\frac{1-y_{2}^{2}}{\left(a^{3}+3 y_{1}\right)^{2 / 3}} \\
& =-\mathrm{N}^{1122}=-\mathrm{N}^{2211}, \\
& \mathrm{~N}^{3131}=\mathrm{N}^{1313}=\left(a^{3}+3 y_{1}\right)\left(a-\left(a^{3}+3 y_{1}\right)^{1 / 3}\right)-\frac{1}{\left(a^{3}+3 y_{1}\right)^{2 / 3}\left(y_{2}^{2}-1\right)} \\
& =-\mathrm{N}^{3311}=-\mathrm{N}^{1133}, \\
& \mathrm{~N}^{4141}=\mathrm{N}^{1414}=\gamma+\left(a^{3}+3 y_{1}\right)\left(\left(a^{3}+3 y_{1}\right)^{1 / 3}-a\right)+2 y_{4} \\
& =-\mathrm{N}^{1144}=-\mathrm{N}^{4411} \text {, } \\
& \mathrm{N}^{5151}=\mathrm{N}^{1515}=\left(a^{3}+3 y_{1}\right)\left(\left(a^{3}+3 y_{1}\right)^{1 / 3}-a\right)+\frac{1}{\gamma+2 y_{4}} \\
& =-\mathrm{N}^{1155}=-\mathrm{N}^{5511}, \\
& \mathrm{~N}^{3232}=\mathrm{N}^{2323}=-\mathrm{N}^{3322}=-\mathrm{N}^{2233}=\frac{y_{2}^{2}\left(y_{2}^{2}-2\right)}{\left(a^{3}+3 y_{1}\right)^{2 / 3}\left(y_{2}^{2}-1\right)}, \\
& \mathrm{N}^{2424}=\mathrm{N}^{4242}=-\mathrm{N}^{2244}=-\mathrm{N}^{4422}=\gamma-\frac{y_{2}^{2}-1}{\left(a^{3}+3 y_{1}\right)^{2 / 3}}+2 y_{4}, \\
& \mathrm{~N}^{2525}=\mathrm{N}^{5252}=-\mathrm{N}^{5522}=-\mathrm{N}^{2255}==\frac{1-y_{2}^{2}}{\left(a^{3}+3 y_{1}\right)^{2 / 3}}+\frac{1}{\gamma+2 y_{4}}, \\
& \mathrm{~N}^{3434}=\mathrm{N}^{4343}=-\mathrm{N}^{3344}=\mathrm{N}^{4433}=-\gamma+\frac{y_{2}^{2}-1}{\left(a^{3}+3 y_{1}\right)^{2 / 3}}-2 y_{4}, \\
& \mathrm{~N}^{3535}=\mathrm{N}^{5353}=-\mathrm{N}^{3355}=-\mathrm{N}^{5533}=-\frac{1}{\left(a^{3}+3 y_{1}\right)^{2 / 3}\left(y_{2}^{2}-1\right)}-\frac{1}{\gamma+2 y_{4}}, \\
& \mathrm{~N}^{4545}=\mathrm{N}^{5454}=-\mathrm{N}^{4455}=-\mathrm{N}^{5544}=-\gamma-2 y_{4}-\frac{1}{\gamma+2 y_{4}} .
\end{aligned}
$$

After some calculations, we obtain the energy and momentum density components of Papapetrou prescription as 


$$
\begin{aligned}
& \Omega^{00}=\frac{1}{8 \pi\left(a^{3}+3 y_{1}\right)^{5 / 3}}\left[\left(a^{3}+3 y_{1}\right)-\frac{a^{2}-2 a\left(\left(a^{3}+3 y_{1}\right)^{1 / 3}-a\right)}{\left(\left(a^{3}+3 y_{1}\right)^{1 / 3}-a\right)^{3}}\right] . \\
& \Omega^{0 i}=0, \quad i=1, \ldots, 5 .
\end{aligned}
$$

The Møller [3] energy-momentum pseudo-tensor $M_{a}^{b}$ is given as

$$
M_{a}^{b}=\frac{1}{8 \pi} K_{a, c}^{b c}
$$

where

$$
K_{a}^{b c}=\sqrt{-g}\left(g_{a d, e}-g_{a e, d}\right) g^{b e} g^{c d} .
$$

Here, $M_{0}^{0}$ stands for the energy density and $M_{i}^{0}(i=1, \ldots 5)$ for the momentum density components. The momentum four-vector is given by

$$
p_{a}=\iint_{V} \int M_{a}^{0} d x^{1} d x^{2} d x^{3}
$$

where $p_{0}$ gives the energy and $p_{a}(a=1, \ldots 5)$ give the momentum. Using Gauss's theorem, the total energy-momentum components may be given in the form of surface integral as

$$
p_{a}=\frac{1}{8 \pi} \int_{S} \int K_{a}^{0 c} \mathbf{n}_{\mathbf{c}} \cdot \mathbf{d} \mathbf{S}
$$

where $\mathbf{n}_{\mathbf{c}}$ is the outward unit normal vector over an infinitesimal surface element $\mathbf{d S}$. When we use Eq.(1) in Eq.(10) the non-vanishing components of $K_{a}^{b c}$, come out as

$$
\begin{aligned}
& K_{01}^{01}=-K^{10}{ }_{0}=a, \\
& K^{12}{ }_{2}=K^{13}{ }_{3}=-K^{21}{ }_{2}=-K^{31}{ }_{3}=2 a-2\left(a^{3}+3 y_{1}\right)^{1 / 3}, \\
& K^{23}{ }_{3}=-K^{32}{ }_{3}=\frac{2 y_{2}}{\left(a^{3}+3 y_{1}\right)^{2 / 3}}, \\
& K^{45}{ }_{5}=-K^{54}{ }_{5}=2 .
\end{aligned}
$$

Making use of these values in Eq.(9), we get the energy-momentum density components of Møller's prescription, which turn out to be zero, i.e.,

$$
\begin{aligned}
M^{00} & =0, \\
M^{0 i} & =0, \quad i=1, \ldots, 5 .
\end{aligned}
$$

\section{Discussion}

The most important issue in GR is an acceptable definition of energy-momentum localization. Although, the problem of localization of energy is unresolved and controversial but numerous scientists attempted to resolve it in the context of different frames of work. For this purpose, a huge number of examples have been explored in different frames of work so that these may help us, at some stage, to make a conjecture about this problem.

It is well-known that geometry and physics are very much related to each other for any physical problem. The motivation behind this work to see what comes out by exploring the energy-momentum distribution of this six-dimensional geometric model of the gravitational field. In other words, to see how is the physics associated with this model. For this purpose, we use Einstein, Landau-Lifshitz, Bergmann-Thomson, Papapetrou and Møller prescriptions. The results obtained are given in this table.

Table 1. Energy Density (ED) Components for all Prescriptions

\begin{tabular}{||c||c||}
\hline \hline ED & Expressions \\
\hline \hline$\Theta^{00}$ & $\frac{-3 y_{1}+a\left(a^{2}+a\left(a^{3}+3 y_{1}\right)^{1 / 3}+\left(a^{3}+3 y_{1}\right)^{2 / 3}\right)}{24 \pi y_{1}\left(a^{3}+3 y_{1}\right)^{2 / 3}}$ \\
\hline \hline$B^{00}$ & $\frac{-3 y_{1}+a\left(a^{2}+a\left(a^{3}+3 y_{1}\right)^{1 / 3}+\left(a^{3}+3 y_{1}\right)^{2 / 3}\right)}{24 \pi y_{1}\left(a^{3}+3 y_{1}\right)^{2 / 3}}$ \\
\hline \hline$L^{00}$ & $\frac{2 a-\left(a^{3}+3 y_{1}\right)^{1 / 3}}{8 a^{3} \pi+24 \pi y_{1}-8 a \pi\left(a^{3}+3 y_{1}\right)^{2 / 3}}$ \\
\hline \hline$\Omega^{00}$ & $\frac{1}{8 \pi\left(a^{3}+3 y_{1}\right)^{5 / 3}}\left[\left(a^{3}+3 y_{1}\right)-\frac{a^{2}}{\left(\left(a^{3}+3 y_{1}\right)^{1 / 3}-a\right)^{3}}-\frac{2 a}{\left(a-\left(a^{3}+3 y_{1}\right)^{1 / 3}\right)^{2}}\right]$ \\
\hline \hline$M^{00}$ & 0 \\
\hline \hline
\end{tabular}


This table shows that the energy density components turn out to be well defined and finite for Einstein, Bergmann-Thomson and Papapetrou prescriptions everywhere in its domain except at $y_{1}=0$ and $y_{1}=-\frac{a^{3}}{3}$ while Papapetrou prescription becomes infinite at $y_{1}=-\frac{a^{3}}{3}$ only. Further, it shows that the results for Einstein and Bergmann-Thomson prescriptions turned out to be same but different from the other prescriptions. It is mentioning here that the energy density becomes zero in case of Møller's prescription. Further, the momentum densities of the six-dimensional geometric model of the gravitational field are zero for all prescriptions. This adds an example of the six-dimensional model towards the solution of the problem of localization of energy and momentum in GR and helpful, at some stage, for making a conjecture about this issue. The graphical representations of the energy density are given as under

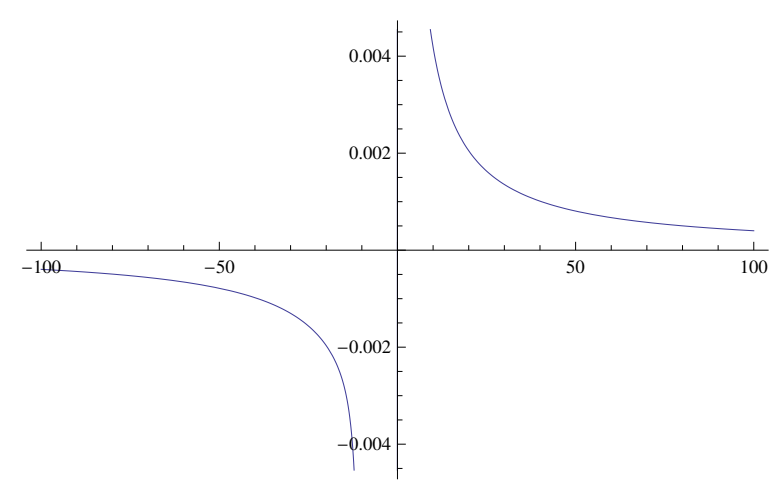

Figure 1: Einstein and Bergmann Thomson Energy density graph vs $y_{1}$

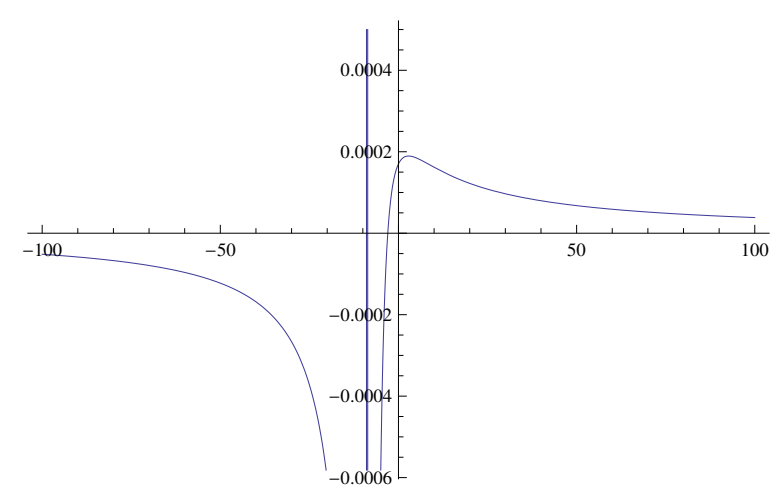

Figure 2: Landau-Lifshitz Energy density graph vs $y_{1}$

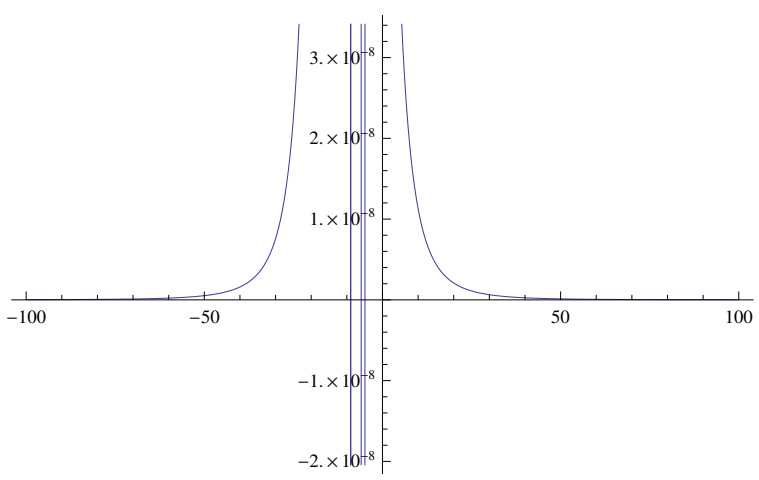

Figure 3: Papapetrou Energy density graph vs $y_{1}$

It is mentioned here that the results of energy-momentum distribution for different spacetimes are not astonishing. On the basis of these results, we can conclude that the energy-momentum complexes, which are treated as pseudo-tensors are not covariant. This is in coherence with the equivalence principle [41] which implies that the gravitational field cannot be detected at a point. The energy-momentum complexes for a huge number of spacetimes have been discussed but no consensus has been built yet to decide which one is the best.

\section{Acknowledgment}

We acknowledge the remarkable assistance of the Higher Education Commission Islamabad, Pakistan, and thankful for its financial support through the Indigenous PhD 5000 Fellowship Program Batch-III. 


\section{References}

[1] A. Einstein, Sitzungsber, On the general theory of relativity, Preus. Akad. Wiss. Berlin (Math. Phys.) 778 (1915).

[2] L. D. Landau, E. M. Lifshitz, The Classical Theory of Fields, (Addison-Wesley Press, New York), (1962).

[3] C. Møller, On the localization of the energy of a physical system in the general theory of relativity, Ann. Phys., 4 (1958), 347.

[4] P. G. Bergmann, R. Thomson, Conservation laws in general relativity as the generators of coordinate transformations, Phys. Rev., 89 (1958), 400.

[5] S. Weinberg, Anthropic bound on the cosmological constant, Phys. Rev. Lett., 59 (1987), 2607.

[6] A. Papapetrou, Einstein's theory of gravitation and flat space, Proc. R. Irish Acad., A52 (1948), 11.

[7] R. C. Tolman, Relativity, Thermodynamics and Cosmology (Oxford University Press, Oxford, 1934).

[8] C. W. Misner, D. H. Sharp, Relativistic Equations for Adiabatic, Spherically Symmetric Gravitational Collapse, Phys. Rev. B, 136 (1964), 571.

[9] F. I. Cooperstock, R. S. Sarracino, The localisation of energy in general relativity, J. Phys. A: Math. Gen., 11 (1978), 877.

[10] K. S. Virbhadra, J. C. Parikh, Gravitational energy of a stringy charged black hole, Phys. Lett., B317 (1993), 312.

[11] K. S. Virbhadra, Naked singularities and Seifert?s conjecture, Phys. Rev., D60(1999), 104041

[12] K. P. Tod, Some examples of Penrose?s quasi-local mass construction, Proc. Roy. Soc., London A388(1983),1795.

[13] R. Penrose, Penrose?s quasi-local mass, Proc. Roy. Soc. London, A381 (1982), 53.

[14] J. W. Maluf, Hamiltonian formulation of the teleparallel description of general relativity, J. Math. Phys., 35 (1994), 335

[15] M. Blagojevic, I. A. Nikolic, Hamiltonian structure of the teleparallel formulation of general relativity, Phys. Rev., D62 (2000), 024021.

[16] J. Schwinger, Quantized gravitational field, Phys. Rev., 130 (1963), 1253.

[17] F. I. Mikhail, M. I. Wanas, A. Hindawi, E. I. Lashin, Energy-momentum complex in Møller's tetrad theory of gravitation, Int. J. Theor. Phys., 32 (1993), 1627.

[18] T. Vargas, The energy of the universe in teleparallel gravity Gen. Rel. Grav., 36 (2004), 1255.

[19] M. Salti, A. Havare, Energy-Momentum in viscous Kasner-type universe in Bergmann-Thomson formulations Int. J. Mod. Phys., A20 (2005), 2169.

[20] M. Salti, Different Approaches for Moller's Energy in the Kasner-type Space-time, Mod. Phys. Lett., A20 (2005), 2175.

[21] M. Salti, Energy?Momentum In The Viscous Kasner-Type Universe In Teleparallel Gravity, Astrophy. Space Sci., 229 (2005), 159.

[22] O. Aydogdu, M. Salti, Energy of the universe in Bianchi-Type I models in Moller's tetrad theory of gravity, Astrophys. Space Sci., 229 (2005), 227.

[23] O. Aydogdu, M. Salti, M. Korunur, Energy in Reboucas-Tiomno-Korotkii-Obukhov and Gödel-type Space-times in Bergmann-Thomson's Formulations, Acta Phys. Slov., 55 (2005), 537.

[24] M. Sharif, M. J. Amir, Teleparallel energy?momentum distribution of lewis-papapetrou spacetimes Mod. Phys. Lett., A22 (2007), 425 .

[25] M. J. Amir, S. Ali, Energy-Momentum Distribution of Non-Static Plane Symmetric Spacetimes in General Relativity and Teleparallel Theory, Chinese Joun. of Phys., $\mathbf{5 0}(2012), 14$.

[26] M. Sharif, M. J. Amir, Teleparallel versions of Friedmann and Lewis-Papapetrou spacetimes, Gen. Relat. Gravit., 38(2006), 1735.

[27] M. Sharif, M. J. Amir, Teleparallel killing vectors of the Einstein universe, Mod. Phys. Lett., A22 (2007), 425.

[28] M. Sharif, M. J. Amir, Teleparallel Version of the Stationary Axisymmetric Solutions and their Energy Contents, Gen. Relat. Gravit., 39 (2007), 989.

[29] M. Sharif, M. J. Amir, Teleparallel Version of the Levi-Civita Vacuum Solutions and their Energy Contents, Canadian J. Phys., 86 (2008), 1091 .

[30] M. Sharif, M. J. Amir, Teleparallel Energy-Momentum Distribution of Static Axially Symmetric Spacetimes, Mod. Phys. Lett., A23 (2008), 3167 .

[31] M. Sharif, M. J. Amir, Energy-Momentum of the Friedmann Models in General Relativity and Teleparallel Theory of Gravity, Canadian J. Phys., 86 (2008), 1297

[32] M. Sharif, M. J. Amir, Teleparallel Energy-Momentum Distribution of Spatially Homogeneous Rotating Spacetimes, Int. J. Theor. Phys., 47 (2008),

[33] T. Multamäki, A. Putaja1, L. Vilja1 , E.C. Vagenas, Energy-momentum complexes in $f(R)$ theories of gravity, Class. Quantum Gravity, 25 (2008), 075017

[34] M. Sharif, M. F. Shamir, Energy distribution in $f(R)$ gravity, Gen. Relativ. Gravit., 42 (2010) 1557.

[35] V. Faraoni, S. Nadeau, The Stability of modified gravity models, Phys. Rev., D72 (2005), 124005.

[36] M. J. Amir, S. Naheed, Spatially Homogeneous Rotating Solution in $f(R)$ Gravity and Its Energy Contents, Int. J. Theor. Phys., 52 (2013), 1688.

[37] M. J. Amir, S. Sattar, Locally Rotationally Symmetric Vacuum Solutions in $f(R)$ Gravity, Int. J. Theor. Phys. 53 (2013), 773.

[38] J. E. G. Silva, V. Santos, C. A. S. Almeida, Gravity localization in a string-cigar braneworld, Class. Quantum Grav., 30 (2013), 025005.

[39] W. D. Linch, G. Tartaglino-Mazzucchelli, Six-dimensional supergravity and projective superfields, JHEP, 08 (2012), 075.

[40] N. Popov, Geometric Model of the Gravitational Field, Gravitation and Cosmology, 4 (1998), 151.

[41] C. W. Misner, K. S. Thorne, J. A. Wheeler, Gravitation, W. H. Freeman and Co., NY 1973.

[42] P. Freud, The energy-momentum problem and the theory of gravitation, Ann. of Math., 40 (1938), 417. 